\title{
Targeting Microglia Using Cx3cr1-Cre Lines: Revisiting the Specificity
}

\author{
Xiao-Feng Zhao, ${ }^{1}$ Mahabub Maraj Alam, ${ }^{1}$ Yuan Liao, ${ }^{3}$ Tingting Huang, ${ }^{1}$ Ramkumar Mathur, ${ }^{3}$ Xinjun \\ $\mathrm{Zhu}^{2}$ and ${ }^{\circ}$ Yunfei Huang ${ }^{1}$
}

https://doi.org/10.1523/ENEURO.0114-19.2019

${ }^{1}$ Department of Neuroscience and Experimental Therapeutics, Albany Medical College, Albany, NY 12208,

${ }^{2}$ Department of Molecular and Cellular Physiology; the IBD Center, Division of Gastroenterology, Department of Medicine, Albany Medical College, Albany, NY 12208, and ${ }^{3}$ Department of Molecular and Cellular Physiology, Albany Medical College, Albany, NY 12208, USA

\begin{abstract}
Microglia play a pivotal role in maintaining homeostasis of the CNS. There is growing interest in understanding how microglia influence normal brain function and disease progression. Several microglia-specific Cx3cr1-Cre lines have been developed and have become indispensable tools in many investigations of microglial function. However, some recent studies have reported that these lines may have significant leakage into neurons. Other studies have reported that $\mathrm{C} \times 3 \mathrm{cr} 1$ is expressed in non-microglial cells, including neurons and astrocytes, in vitro or in vivo either during brain development or upon neurological insult. All these reports raise serious concerns about the trustworthiness of these Cre-lines and whether the conclusions drawn from previous studies are valid. Here, we found that a floxed fluorescent reporter mouse line which has been frequently used to verify Cre lines displayed spontaneous expression of the GFP reporter, independent of Cre recombinase, thus revealing a potential caveat in assessing cre lines. We further confirmed that two Cx3cr1-Cre mouse lines can drive fluorescent reporter expression largely restrictively in microglia. Finally, we clarified that these two mouse lines maintain microgliaspecific expression even following excitatory injury. Together, our findings confirm that two previously created Cx3cr1-Cre lines remain as invaluable tools for studying microglia. Moreover, to ensure the quality of data generated and the soundness of conclusions drawn from such data, it should be compulsory to thoroughly examine reporter lines for spontaneous leakiness when labeling cells to study CNS function and diseases.
\end{abstract}

Key words: cre; cx3cr1; epilepsy; microglia; reporter; tracing

\section{Significance Statement}

Microglia-specific Cre-lines are essential for studying the role of microglia in the CNS. Several Cx3cr1-Cre lines have been developed and used in a number of landmark studies. However, there is growing concern in the microglia research community regarding potential leakiness of Cre-lines into neurons. The conclusions drawn from previous studies are also being questioned and key ongoing studies have been stalled. We found that a GFP reporter mouse lines used in a previous study displays spontaneous leakiness into neurons, independent of Cre recombinase. Furthermore, we confirmed that two Cre-lines are microgliaspecific and thus can be redeployed without hesitation. Our study also suggests that testing for potential leakiness of GFP reporter lines should be included as a control in cell-tracing experiments.

Received March 18, 2019; accepted May 28, 2019; First published June 14, 2019.

The authors declare no competing financial interests.
Author contributions: X.-F.Z. and Y.H. designed research; X.-F.Z. performed research; X.-F.Z., M.M.A., Y.L., R.M., T.H., and X.Z. contributed unpublished reagents/analytic tools; X.-F.Z. analyzed data; X.-F.Z. and Y.H. wrote the paper. 


\section{Introduction}

Microglia are present nearly uniformly throughout the entire central nervous system, where they constantly prune synapses and repair minor injuries. Microglia are also the chief resident immune cells, part of the innate immune response to "danger" signals. Genetic tools that specifically target microglia are essential for unlocking the roles of microglia in the CNS and neurologic diseases.

Endogenous $\mathrm{C} \times 3 \mathrm{cr} 1$ is a selective marker for microglia in the CNS, whereas its ligand fractalkine is expressed in neurons. Several mouse lines have been created for studying microglia (Wieghofer and Prinz, 2016). The Cx3cr1-GFP reporter line (stock \#005582; The Jackson Laboratory) was created by replacing the coding part of the Cx3cr1 gene with green fluorescent protein (GFP; Jung et al., 2000). This reporter line has been widely used to label microglia for visualization. The expression pattern of Cx3cr1-driven GFP reporter is specific to microglia over the entire period of postnatal brain development and appears to be maintained even following an excitatory injury (Kim et al., 2015). Using a BAC transgenic strategy, two constitutive and two inducible $\mathrm{C} \times 3 \mathrm{cr} 1$ promoterdriving cre lines were created (Gong et al., 2003, 2007; Parkhurst et al., 2013; Yona et al., 2013; Peng et al., 2016). The constitutive Cx3cr1-cre line created by Jung et al. (2000; stock \#025524; The Jackson Laboratory) was recently reported to have significant leakage into neurons (Haimon et al., 2018; Zhang et al., 2018). Another constitutive Cx3cr1-Cre line was developed by the Mutant Mouse Resource and Research Center (MMRRC). This line was reported to drive microglial-specific expression of a reporter (also created by MMRRC) in the majority of animals examined (Hwang et al., 2017). However, 10\% of the animals displayed significant leakage into neurons (Hwang et al., 2017). In another study, this line was reported to drive the expression of $\mathrm{mT} / \mathrm{mG}$ reporter (stock \#007676; The Jackson Laboratory) selectively in microglia (Zhao et al., 2018). Two Cx3cr1-CreERT2 inducible lines were created separately by two laboratories. In initial studies, these lines were found to deliver microglialspecific expression (Parkhurst et al., 2013; Yona et al., 2013). However, a recent study found that one of these inducible lines displays significant leakage of the RosaGreen reporter (stock \#007906; The Jackson Laboratory) into neurons (Zhang et al., 2018). It is unclear why the leakage into neurons varies among the different Cx3cr1cre lines and studies (Parkhurst et al., 2013; Hwang et al., 2017; Zhang et al., 2018; Zhao et al., 2018), but the problem has raised concerns about whether there is a reliable cre-line that targets microglia with good specificity. Moreover, investigators now hesitate to use these

This work was supported by the National Institutes of Health Grant NS093045 (to Y.H.).

Correspondence should be addressed to Yunfei Huang at huangy@amc.edu.

https://doi.org/10.1523/ENEURO.0114-19.2019

Copyright (C) 2019 Zhao et al.

This is an open-access article distributed under the terms of the Creative Commons Attribution 4.0 International license, which permits unrestricted use, distribution and reproduction in any medium provided that the original work is properly attributed. lines and some have questioned the validity of the data generated in the previous studies (Parkhurst et al., 2013; Hwang et al., 2017; Zhang et al., 2018; Zhao et al., 2018). Lack of validated cre lines that specifically target microglia would be a significant setback to the field. Therefore, it is imperative to understand how the variable leakiness among these lines is occurring and how it might be rectified. Here, we performed a thorough characterization of three popular GFP reporter lines, along with two Cx3cr1 promoter-driven Cre lines.

\section{Materials and Methods}

\section{Animals}

Male and female mice were housed in a pathogenfree, temperature-controlled, and humidity-controlled facility with a 12/12 h light/dark cycle (lights on at 7 A.M.) and given ad libitum access to food and water. All experiments were performed according to the guidelines set by the Institutional Animal Care and Use Committee as well as the National Institutes of Health Guide for the Care and Use of Laboratory Animals. B6.CgGt(ROSA)26Sor ${ }^{t m 6(C A G-Z s G r e e n 1) H z e / J}$ (hereafter referred to as Rosa-Green ${ }^{f / f}$; stock \#007906), B6.CgGt(ROSA)26Sor ${ }^{\text {tm14(CAG-tdTomato)Hze/J }}$ (hereafter referred to as TdTomato ${ }^{\mathrm{f} / \mathrm{f}}$; stock \#007914), B6.129(Cg)Gt(ROSA)26Sor tm4(ACTB-tdTomato,-EGFP)Luo/J (hereafter referred to as $\mathrm{mT} / \mathrm{mG}^{\mathrm{f} / \mathrm{f}}$; stock \#007676), and B6.129P2(C)-Cx3cr1 tm2.1(cre/ERT2)Jung/J (hereafter referred to as Cx3cr1-CreERT2; stock \#020940) were acquired from The Jackson Laboratory. Tg(Cx3cr1Cre)MW126Gsat/Mmucd (hereafter referred to as Cx3cr1-CreM; stock \#036395-UCD) mice were acquired from the MMRRC (Table 1).

\section{Antibodies, immunohistochemistry (IHC), and acquisition of images}

Male and female mice were anesthetized with pentobarbital (100 mg/kg, i.p.) and transcardially perfused with PBS followed by $4 \%$ paraformaldehyde (PFA) in PBS, pH 7.4. Brains were post-fixed overnight in $4 \%$ PFA buffer, followed by cryoprotection in $30 \%$ sucrose in PBS for at least $48 \mathrm{~h}$. Mouse brains were then embedded in Neg-50 frozen section medium (Fisher Scientific) and sectioned using a cryostat at $35 \mu \mathrm{m}$ for all histologic analyses. Brain coronal sections with similar anatomic locations (near bregma $-2 \mathrm{~mm}$ position based on the mouse brain atlas) were selected for all histologic analyses. Brain sections were washed with PBS, blocked and permeabilized with 10\% BSA (Sigma) and 0.3\% Triton X-100 (Sigma) in PBS at room temperature for $1 \mathrm{~h}$. Brains were stained with rabbit anti-GFP (1:2000; catalog \#ab290; Abcam), rabbit anti-lba1 (1:400; catalog \#019-19471; Wako), rabbit antiNeuN (1:400; catalog \#24307; Cell Signaling Technology; Subbanna et al., 2018), mouse anti-NeuN (1:250; catalog \#MAB377; Millipore; Sahay et al., 2011), and rabbit antiGFAP (1:500; catalog \#AB5541; Millipore) (Table 1). Sections were incubated with primary antibodies overnight at $4^{\circ} \mathrm{C}$, and then washed with PBS for $5 \mathrm{~min}$ and repeated three times, followed by incubation with appropriate fluorescent-conjugated secondary antibodies for $2 \mathrm{~h}$ at 
Table 1. Materials and reagents

\begin{tabular}{|c|c|c|c|}
\hline Reagent or resource & Source & Catalog number & RRID \\
\hline \multicolumn{4}{|l|}{ Antibodies } \\
\hline Goat polyclonal anti lba1 & Novus Biologicals & NB100-1028 & AB_521594 \\
\hline Rabbit anti-NeuN & Cell Signaling & 24307 & AB_2651140 \\
\hline Chicken anti-glial fibrillary acidic protein & Millipore & AB5541 & $A B_{-}+177521$ \\
\hline $\begin{array}{l}\text { Rabbit anti-GFP } \\
\text { chemicals }\end{array}$ & Abcam & ab290 & AB_303395 \\
\hline$(-)$-Scopolamine methyl bromide & Sigma & S8502 & $\mathrm{N} / \mathrm{A}$ \\
\hline Lectin-DyLight 488 & Vector Laboratories & DL-1174 & AB_2336404 \\
\hline DAPI & Sigma & D9542 & $\mathrm{N} / \mathrm{A}^{-}$ \\
\hline \multicolumn{4}{|l|}{ Experimental models: organisms/strains } \\
\hline ROSA-Green mice & The Jackson Laboratory & 007906 & IMSR_JAX:007906 \\
\hline $\mathrm{mT} / \mathrm{mG}$ mice & The Jackson Laboratory & 007676 & IMSR_JAX:007676 \\
\hline Fiji-ImageJ & Fiji & $\begin{array}{l}\text { https://fiji.sc/ or } \\
\text { https://imagej.nih.gov/ij/ }\end{array}$ & SCR_002285 \\
\hline GraphPad Prism 7.0 & GraphPad Software & https://www.graphpad.com & SCR_002798 \\
\hline Zen Black & Zeiss & https://www.zeiss.com & $\mathrm{N} / \mathrm{A}$ \\
\hline Zen Blue & Zeiss & https://www.zeiss.com & SCR_013672 \\
\hline Neurolucida & MBF Bioscience & http://www.mbfbioscience.com & SCR_001775 \\
\hline
\end{tabular}

The source, category, and PRID number of antibodies, chemicals, and mouse lines were provided.

room temperature. For brain sections that needed to be stained with goat anti-Iba1 (1:250; catalog \#NB100-1028; Novus Biologicals), free-floating sections were permeabilized and blocked with blocking buffer containing $0.3 \%$ Triton X-100 and 5\% normal donkey serum in PBS at room temperature for $1 \mathrm{~h}$. Sections were then incubated with goat anti-lba1 diluted in blocking buffer overnight at $4^{\circ} \mathrm{C}$, and then washed with PBS for $5 \mathrm{~min}$ and repeated three times, followed by incubation with appropriate fluorescent-conjugated secondary antibodies diluted in blocking buffer for $2 \mathrm{~h}$ at room temperature. Nuclei were counterstained with DAPI (Sigma) and coverslips were applied with Fluoromount G (Southern Biotech) and sealed with nail polish. Co-immunostaining requires the consideration of the compatibility of antibody host species. Therefore, two pairs of anti-lba1 and anti-NeuN antibodies have been used to detect microglia and neurons, respectively, in the present study and explicitly noted in the figure legends. Both pairs of antibodies are cell-type specific (Extended Data Fig. 1-3A,B ). Lycopersicon esculentum lectin was used to label the endothelial cells of the blood vessels (Mazzetti et al., 2004). All representative images were acquired using a Zeiss LSM 880 confocal microscope with Airyscan and processed with Zen black 2.1 or Zen blue lite 2.3 (Carl Zeiss). Images were acquired using the tiles and positions module and a $25 \times$ water objective lens. Each tile represents a z-stack of 15 images at $1-\mu \mathrm{m}$ intervals acquired from a single position, and images (tiles) from a series of positions were stitched together, followed by maximal intensity projection to form a 2-D image as presented. For quantification of Iba1positive, NeuN-positive, and GFAP-positive cells, a full mosaic of entire brain sections was acquired using a
Neurolucida imaging system (MBF Bioscience), under a $10 \times$ lens and quantified with Neurolucida software. Microglia within the areas of the M1 motor cortex around Layer IV, the hippocampal radiatum layer adjacent to pyramidal CA1, the stratum lucidum adjacent to CA3, and the dentate gyrus (hereafter referred to as cortex, CA1, $\mathrm{CA} 3$, and DG, respectively), were quantified. $\mathrm{GFP}^{+} / \mathrm{Iba}^{+}$, $\mathrm{GFP}^{+} / \mathrm{NeuN}^{+}$, and $\mathrm{GFP}^{+} / \mathrm{GFAP}^{+}$double-positive cells over the entire brain sections were quantified.

\section{Tamoxifen treatment}

Tamoxifen (catalog \#T5648; Sigma-Aldrich) was dissolved in corn oil (catalog \#C8267; Sigma-Aldrich) at a concentration of $20 \mathrm{mg} / \mathrm{ml}$ by shaking overnight at $37^{\circ} \mathrm{C}$. Dissolved tamoxifen was stored at $4^{\circ} \mathrm{C}$ for the duration of injections. Cx3cr1-CreERT2J $\pm ; \mathrm{mT} / \mathrm{mG}^{\mathrm{f} /}$ mice seven to eight weeks old (either sex) were injected intraperitoneally with tamoxifen daily at $100 \mathrm{mg} / \mathrm{kg}$ body weight for five consecutive days.

\section{Pilocarpine treatment}

Status epilepticus (SE) was induced by pilocarpine as described in previous studies (Müller et al., 2009). In brief, to minimize the peripheral cholinergic side effects of pilocarpine, 8- to 10-week-old mice of either sex were first injected intraperitoneally with $1 \mathrm{mg} / \mathrm{kg}$ methyl scopolamine (S8502; Sigma-Aldrich) in $0.9 \% \mathrm{NaCl}$ for $10 \mathrm{~min}$ before injection of pilocarpine (P6503; Sigma-Aldrich). For all groups, pilocarpine administration was started at the same time of day, 9 A.M. to 3 P.M. We used a modified ramping-up pilocarpine injection protocol (Müller et al., 2009). In brief, the first dose of $200 \mathrm{mg} / \mathrm{kg}$ pilocarpine was given to all the mice by intraperitoneal injection and fol- 
lowed by repeated low-dose treatment at $50 \mathrm{mg} / \mathrm{kg}$ every 15 min until the onset of stage four or five seizures. Seizures were classified according to the Racine scale (Racine, 1972), with modification made by Borges et al. (2003): stage 0, normal activity; stage 1, rigid posture or immobility; stage 2, stiffened, extended and often arched tail; stage 3 , partial body clonus, including forelimb or hindlimb clonus or head bobbing; stage 4 , whole body continuous clonic seizures with rearing; stage 5, severe whole-body continuous clonic seizures with rearing and falling; stage 6 , tonic-clonic seizures with loss of posture or jumping. Animals were allowed to develop SE for $4 \mathrm{~h}$. SE was terminated by diazepam treatment $(10 \mathrm{mg} / \mathrm{kg}$, i.p.), followed by administration of a single dose of dextrose $\left(1.5 \mathrm{~g} / \mathrm{kg}\right.$, i.p.). Animals were placed on a $30^{\circ} \mathrm{C}$ warm pad for recovery for $1 \mathrm{~h}$.

\section{Statistical analysis}

Data were analyzed using GraphPad Prism 7 software and presented as the mean \pm SEM.

\section{Results}

\section{Spontaneous leakiness of Cre-dependent GFP reporter lines in the CNS}

GFP reporter lines are frequently used to verify the cell specificity of Cre lines. While Cre lines have been blamed for the cause of leakiness into neurons, the reliability of Cre-dependent GFP reporter lines has not been seriously evaluated or even discussed. We started by examining the Rosa-Green ${ }^{f / f}$ reporter line (stock \#007906), which has been widely used. This reporter line was recently used to evaluate the microglial specificity of the constitutive B6J.B6N(Cg)-Cx3cr1 ${ }^{\text {tm1.1(cre)Jung/J }}$ (Cx3cr1-CreJ; stock \#025524; The Jackson Laboratory) and inducible B6.129P-Cx3cr1 ${ }^{\text {tm1Litt/J }}$ (Cx3cr1-CreERT2, stock\#: 005582; The Jackson Laboratory) Cre lines (Zhang et al., 2018). In that study, substantial neuronal leakiness of the GFP reporter was reported. We harvested Rosa-Green ${ }^{f / f}$ mouse brains at around two to six months of age and selected three sections from anatomically comparable regions for evaluation. To our surprise, $33.33 \%$ of the animals displayed significant expression of GFP reporter throughout the brain, from individually sporadic GFPpositive cells to clusters (Fig. 1A,C; Extended Data Fig. $1-1 A$ ). The leakiness varied significantly between the two hemispheres (Extended Data Fig. 1-1A) and among animals even in the same age group. Among the GFPpositive cells in the cortex, lba1 ${ }^{+}, \mathrm{NeuN}^{+}$, and $\mathrm{GFAP}^{+}$ populations are $2.80 \pm 2.45 \%, 17.42 \pm 15.66 \%$, and $0 \%$, respectively. In the hippocampus, Iba1+, $\mathrm{NeuN}+$, and GFAP + populations are $0 \%, 41.71 \pm 15.45 \%$, and $0.47 \pm$ $0.83 \%$, respectively. Therefore, a significant portion of GFP-positive cells are lba1/NeuN/GFAP- triple negative in the cortex $(79.8 \%)$ and hippocampus $(57.8 \%$; two males and one female). Notably, some weak NeuN-positive cells lacked typical neuronal morphology (Fig. 1C; Extended Data Fig. 2-1B,C). the exact identity of these cells needs to be determined.

The tdTomato line is also frequently used for cell lineage tracing. According to The Jackson Laboratory web- site, this line may express very low levels of red fluorescent protein (tdTomato) in the absence of cre recombinase. In addition, a very small portion of the animals was found to be very leaky, leading to the whole body becoming pinkish. Accordingly, we evaluated this reporter line. We found that the seven animals examined display significant leakage of tdTomato in the brain (four males and three females; Fig. 1B,D). Among the tdTomato positive cells in the cortex, $\mathrm{Iba1}^{+}, \mathrm{NeuN}^{+}$, and $\mathrm{GFAP}^{+}$populations are $2.00 \pm 4.5 \%, 4.00 \pm 8.9 \%$, and $0 \%$, respectively. In the hippocampus, Iba1,$+ \mathrm{NeuN}+$, and GFAP + populations are $0,44 \pm 0.9 \%, 0.70 \pm 1.6 \%$, and $0.88 \pm 1.2 \%$, respectively. It appears that tdTomato predominantly labels vascular structures in the CNS. Accordingly, we employed lycopersicon esculentum lectin (Mazzetti et al., 2004) to label endothelial cells in the blood vessels (Extended Data Fig. 1-2A,B). We observed that tdTomato signal is co-localized with lectin, particularly in the hippocampus. This data suggests that there is a significant leakage of tdTomato expression into the blood vessels in the CNS in the absence of cre recombinase.

The $\mathrm{mT} / \mathrm{mG}$ reporter line is most frequently used in cell lineage tracing. Mice are expected to express membrane tagged-Tomato (mTomato) ubiquitously independent of cre recombinase whereas the expression of membrane tagged-GFP(mGFP) requires cre-mediated homologous recombination. Indeed, mTomato is expressed in various brain structures but is noticeably present in the hippocampal CA3 area and in the blood vessels (Extended Data Fig. $1-2 C, D)$. We saw that lectin is colocalized with mTomato, confirming that mTomato is highly expressed in the blood vessels. We employed an anti-GFP antibody to detect the expression of mGFP. We found no leakage of mGFP expression over the entirety of the sections from all seven $\mathrm{mT} / \mathrm{mG}^{\mathrm{f} / \mathrm{f}}$ mice we examined (three males and four females; Fig. 1E,F; Extended Data Fig. 1-1B). Therefore, we conclude that the $\mathrm{mT}_{/} \mathrm{mG}^{\mathrm{f} / \mathrm{f}}$ line is a reliable reporter for evaluating Cre lines in the CNS.

\section{The Cx3cr1-creM mouse line mediates microglial expression of floxed GFP reporters}

Heterozygous $\mathrm{Cx} 3 \mathrm{cr} 1-\mathrm{CreM} \pm$ mice were acquired from the MMRRC. This line has been maintained in a heterozygous state by crossing with wild-type mice. We mated Cx3cr1-CreM \pm mice with mice homozygous for the Rosa-Green $^{f / f}$ reporter to generate Cx3cr1-CreM \pm ;Rosa$\mathrm{Green}^{\mathrm{f} / \mathrm{-}}$ mice. Brains were harvested from the latter at around two months of age. We found that $99.2 \pm 0.3 \%$ and $99.8 \pm 0.16 \%$ of microglia are GFP-positive in the cortex and hippocampus, respectively (two males and three females; Fig. $2 A-D$ ). However, we also observed several dozens of non-microglial GFP-positive cells scattered sporadically throughout the entire brain (Fig. 2A-D; Extended Data Fig. 2-1A-C). $\mathrm{NeuN}^{+} / \mathrm{GFP}^{+}$and $\mathrm{GFAP}^{+} /$ $\mathrm{GFP}^{+}$populations are $0.13+0.5 \%$ and $0 \%$, in the cortex, and $0 \%$ and $0.2 \pm 0.5 \%$ in the hippocampus, respectively. These non-microglial GFP-positive cells likely result from leakage of the Rosa-Green reporter itself. The specificity of the Cx3cr1-CreM line was further verified in Cx3cr1-Cre $\pm ; \mathrm{mT} / \mathrm{mG}^{\mathrm{f} /-}$ mice (Fig. 2E-J). We observed 
A Rosa-Green ${ }^{f / f}$, The Jackson Laboratory, Stock\#007906
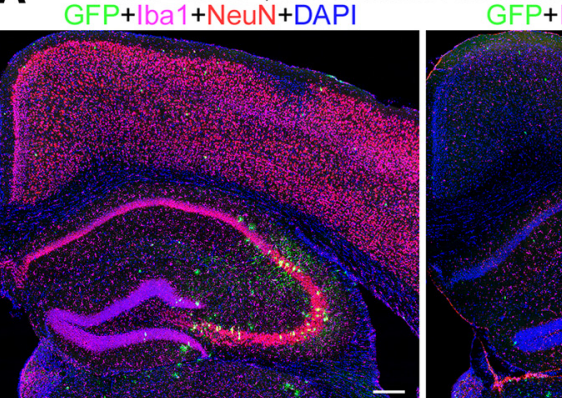

B tdTomato $f / f$, The Jackson Laboratory, Stock\#007914

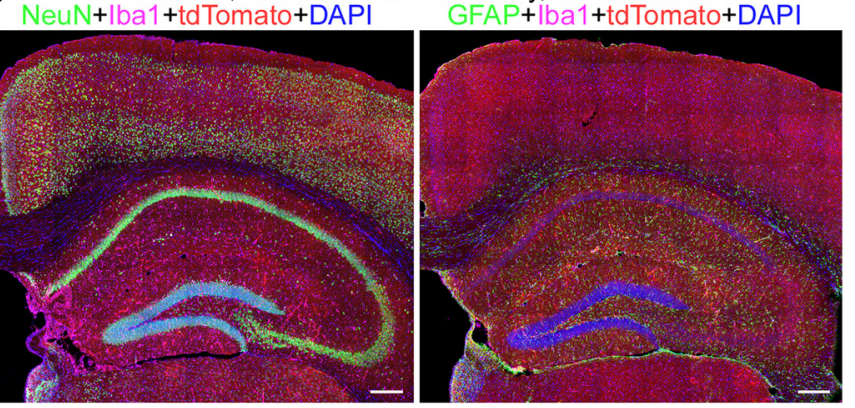

C Rosa-Green ${ }^{f / f}$, The Jackson Laboratory, Stock\#007906

D
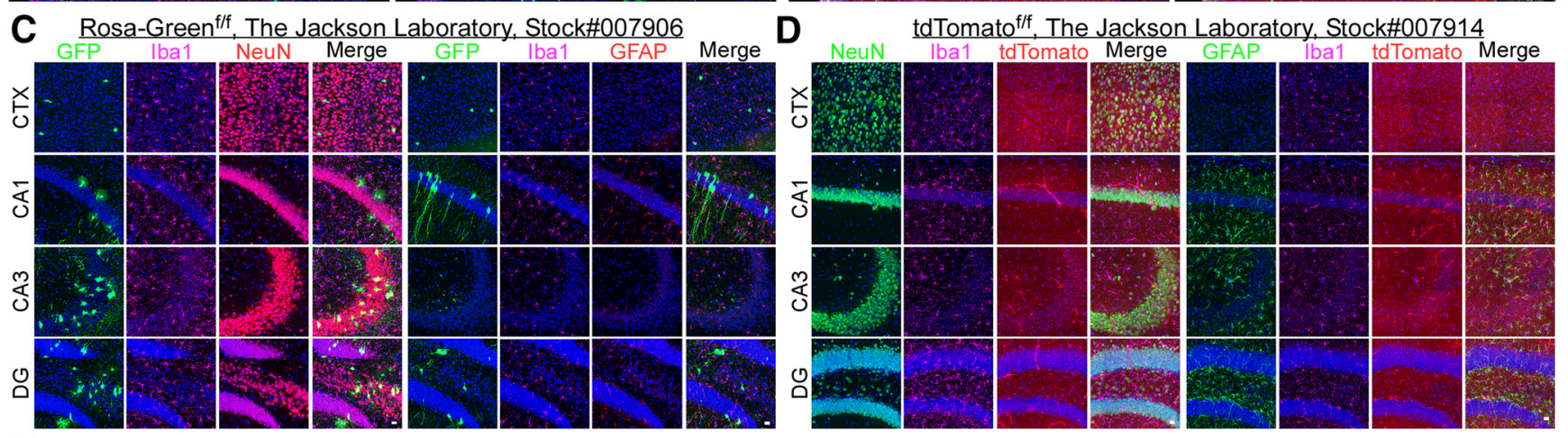

E

$\mathrm{mT} / \mathrm{mG}^{\mathrm{f} / \mathrm{f}}$, The Jackson Laboratory, Stock\#007914
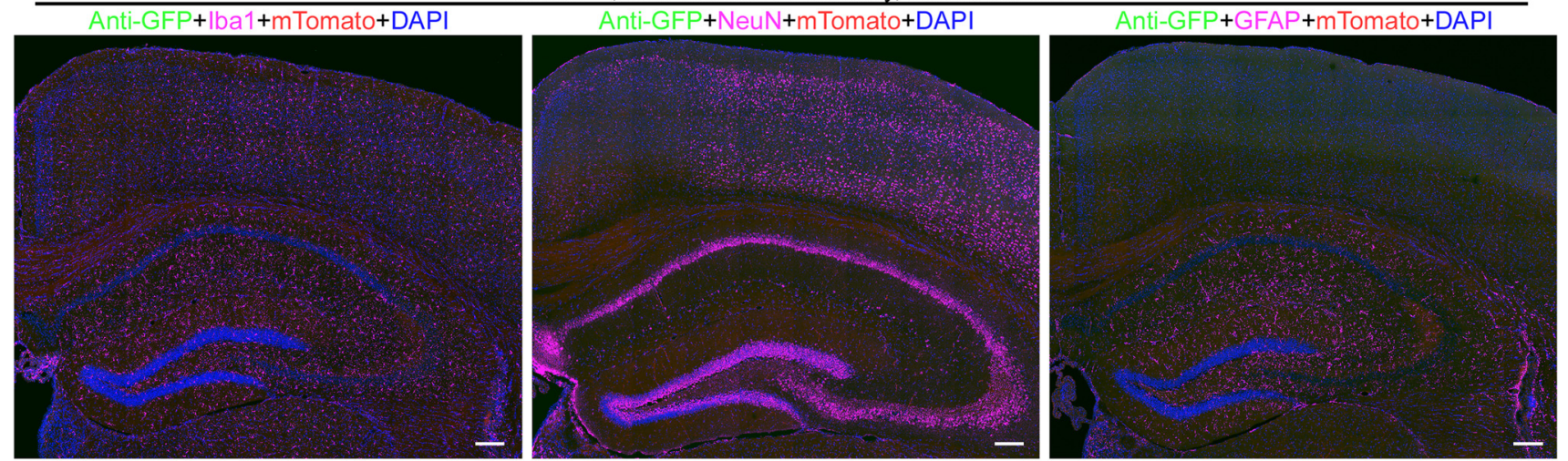

$\mathbf{F}$

$\mathrm{mT} / \mathrm{mG}^{\mathrm{f} / \mathrm{f}}$, The Jackson Laboratory, Stock\#007914
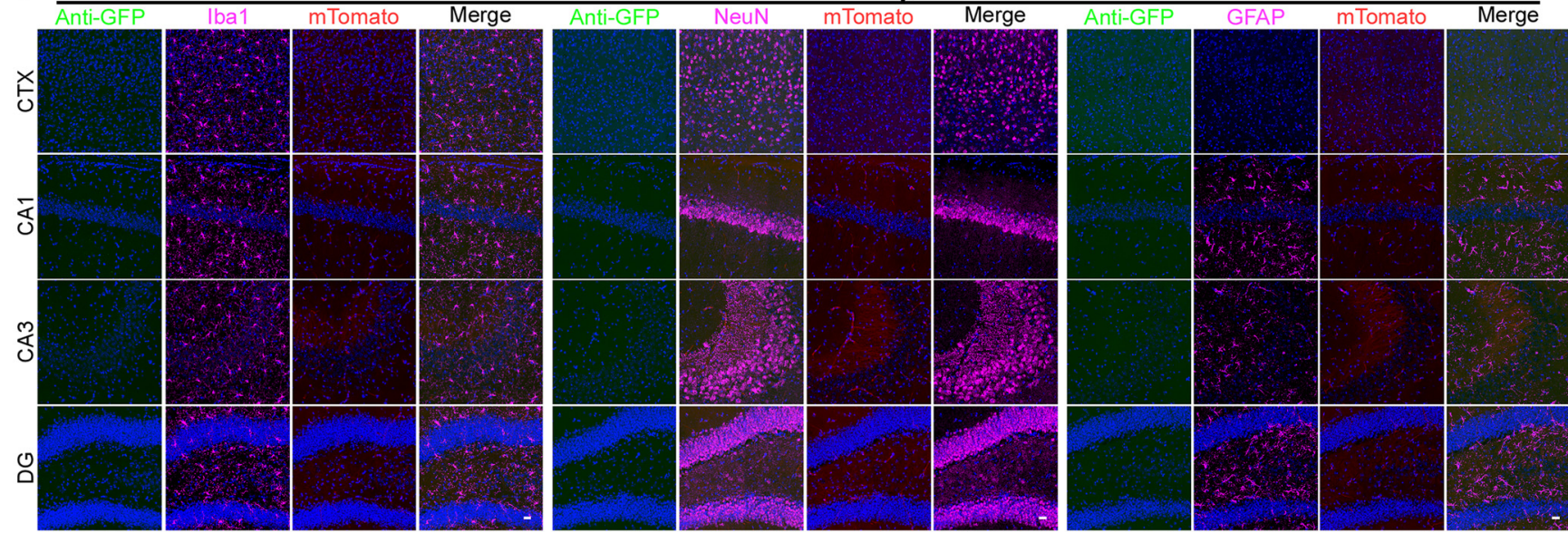

Figure 1. Leakage of fluorescent reporter lines. A, Full montage of confocal images showing GFP expression in the cortex and hippocampus of the Rosa-Green ${ }^{\mathrm{f} / \mathrm{f}}$ mouse line. Mouse brain sections were stained with: goat anti-lba1 (pink), rabbit anti-NeuN (red) or chicken anti-GFAP (red), and DAPI (blue). Scale bar, $200 \mu \mathrm{m}$. B. Full montage of images showing tdTomato expression in the cortex and hippocampus of the tdTomato ${ }^{f / f}$ mouse line. Mouse brain sections were stained with: rabbit anti-lba1 (pink), mouse anti-NeuN (green) or chicken anti-GFAP (green), and DAPI (blue). Scale bar, $200 \mu \mathrm{m}$. C, High-magnification images showing that the morphology 
continued

of GFP-positive cells varies among anatomic regions in the cortex (CTX) and the hippocampal CA1, CA3, and DG in Rosa-Green ${ }^{\mathrm{f} / \mathrm{f}}$ mice. Scale bar, $20 \mu \mathrm{m}$. D. High-magnification images showing tdTomato-positive cells in the CTX and the hippocampal CA1, CA3,

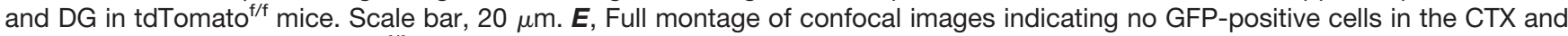
the hippocampus of the $\mathrm{mT} / \mathrm{mG}^{\mathrm{f} / \mathrm{f}}$ mouse line. Mouse brain sections were stained with: rabbit anti-GFP (green), goat anti-lba1 (pink) or mouse anti-NeuN (pink) or chicken anti-GFAP (pink), and DAPI (blue). Scale bar, $200 \mu \mathrm{m}$. $\boldsymbol{F}$, High-magnification images no

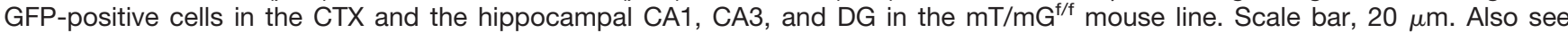
Extended Data Fig. 1-1, Fig. 1-2, Fig.1-3.

that among the GFP-positive cells, $99.9 \pm 0.17 \%$ and $99.7 \pm 0.36 \%$ were lba1 positive in the cortex and hippocampus, respectively (Fig. 2E,G); none of $\mathrm{GFP}^{+}$cells are either NeuN-positive or GFAP-positive (two males and two females; Fig. $2 F, H-J)$. Taken together, these data suggest that the $\mathrm{Cx} 3 \mathrm{cr} 1-\mathrm{CreM} \pm$ line drives microglialspecific expression of floxed GFP reporters.

\section{Leakiness of the inducible Cx3cr1-creERT2 mouse line}

A recent study reported that the Cx3cr1-CreERT2 line (Parkhurst et al., 2013) has a significant leakage into neurons (Zhang et al., 2018). Another Cx3cr1-CreERT2 mouse line was developed by Jung et al. (2000). This prompted us to determine whether this line, too, has any leakiness into neurons. We crossed this inducible line with an $\mathrm{mT} / \mathrm{mG}^{\mathrm{f} / \mathrm{f}}$ reporter line and mGFP expression was detected by anti-GFP antibody. We found that Cx3cr1CreERT2J $\pm ; \mathrm{mT} \mathrm{mG}^{\mathrm{f} /}$ - mice displayed significant expression of GFP reporter in microglia even without tamoxifen treatment (one male and two females; Fig. $3 A-F$ ). In vehicle treated mice, up to $18.9 \pm 4.3 \%$ and $23.0 \pm 3.4 \%$ of Iba $1^{+}$cells are GFP positive in the cortex and hippocampus, respectively. After tomaxifen treatment, all $\mathrm{lba} 1^{+}$ cells are GFP positive (two males and one female). Again, none of GFP positive cells are either NeuN- or GFAPpositive. These data suggest that in this Cx3cr1CreERT2J mouse line, Cre-ERT2 recombinase possesses some basal enzymatic activity independent of tamoxifen. However, the Cre-dependent expression is nearly completely restricted to microglia (Fig. $3 A-F$ ).

\section{Transient Cx3cr1 activity in neurons and astrocytes following excitatory injury}

Cx3cr1 was reported to be expressed in cultured neurons and induced in neurons and astrocytes following brain ischemia and severe seizures, respectively (Meucci et al., 2000; Yeo et al., 2011; Dworzak et al., 2015; Wang et al., 2018). It was postulated that leakiness into neurons was due to transient expression of Cx3cr1. However, there is no reliable anti-Cx3cr1 antibody available. It is unclear whether the transient induction of Cx3cr1 reported in neurons and astrocytes is real. Moreover, the Cx3cr1-GFP mouse line did not show any leakage of the GFP reporter into neurons and astrocytes under excitatory conditions (Kim et al., 2015). Nevertheless, we evaluated transient $\mathrm{C} \times 3 \mathrm{cr} 1$ activity in $\mathrm{C} \times 3 \mathrm{cr} 1-\mathrm{CreM} \pm$; RosaGreen $^{\mathrm{t} /-}$ (Fig. 4A-D) and $\mathrm{C} \times 3 \mathrm{cr} 1-\mathrm{CreM} \pm ; \mathrm{mT}^{-} \mathrm{mG}^{\mathrm{t} /}$-mice (Fig. 4E-H). Animals around eight weeks of age were treated with pilocarpine to induce SE for $4 \mathrm{~h}$. Mouse brains were harvested 3 d post-SE. In $\mathrm{C} \times 3 \mathrm{cr} 1-\mathrm{CreM} \pm$;
Rosa-Green ${ }^{\mathrm{f} /}$, we found that $98.8 \pm 1.1 \%$ and $99.6 \pm$ $0.24 \%$ of $\mathrm{GFP}^{+}$cells were lba1-positive in the cortex and hippocampus, respectively (two males and one female). Among the other $\mathrm{GFP}^{+}$cells, only $0.31 \pm 0.54 \%$ and 0.07 $\pm 0.13 \%$ are NeuN positive in the cortex and hippocampus, respectively and none of them are GFAP-positive (Fig. 4A-D). Those non-microglial GFP-positive cells are presumably from the spontaneous leakiness of the Rosa$\mathrm{Green}^{\mathrm{f} / \mathrm{f}}$ reporter. Moreover, in Cx3cr1-Cre $\pm ; \mathrm{mT} / \mathrm{mG}^{\mathrm{f} /-}$ mice, we observed that nearly all GFP-positive cells were microglia $(99.9 \pm 0.13 \%$ in the cortex and $100 \%$ in the hippocampus; two males and one female; Fig. $4 E-H)$. We saw few, if any, $\mathrm{NeuN}^{+} / \mathrm{GFP}^{+}$or $\mathrm{GFAP}^{+} / \mathrm{GFP}^{+}$doublepositive cells. Thus, our data confirm there is little or no transient Cx3cr1-driven Cre activity in neurons or astrocytes in brains that experienced SE.

\section{Discussion}

A reliable microglial-specific Cre line is essential for elucidating the role of microglia in normal CNS function and neurologic disorders. Over the past four to five years, multiple Cx3cr1-Cre lines have been developed for targeting microglia. Unfortunately, leakiness of cre activity into neurons has become a major concern (Zhang et al., 2018). Leakiness is a confounding factor that significantly limits the usefulness and interpretation of the data generated, and greatly discourages the use of these lines. In the present study, we unexpectedly found that the RosaGreen $^{\mathrm{f} / \mathrm{f}}$ reporter line used in a previous study by other researchers (Zhang et al., 2018) shows spontaneous leakiness into non-microglial cells in the absence of cre recombinase. This cre-independent leakage likely accounts, at least in part, for the GFP-positive nonmicroglial cells observed in that previous study (Zhang et al., 2018). We have also confirmed that the Cx3cr1CreM line from MMRRC is reasonably microglia specific. The inducible Cx3cr1-CreERT2 line developed by Jung et al. (2000), displayed some basal cre activity without tamoxifen treatment, but this expression was restricted to microglia. Finally, we did not find any significant transient Cx3cr 1 activity in neurons and astrocytes under the conditions that animals have experienced excitatory injury.

Many cre-dependent fluorescence-based reporter lines have been created for cell-lineage tracing. These reporter lines are also frequently used to evaluate the specificity and recombination efficiency of Cre lines. One would naturally expect that the floxed-fluorescecnt reporter lines are cre dependent. Surprisingly, however, the Rosa$\mathrm{Green}^{\mathrm{f} / \mathrm{f}}$ and tdTomato $\mathrm{t}^{\mathrm{f} / \mathrm{f}}$ lines displayed spontaneous leakiness independent of cre recombinase. Our study suggests that the Rosa-Green ${ }^{f / f}$ reporter line itself could 
A
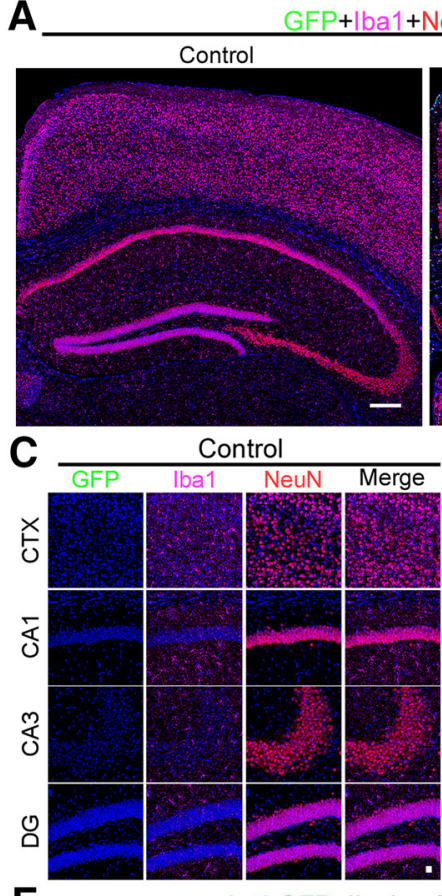

E

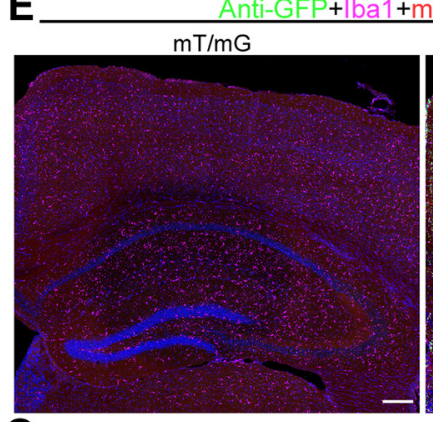

G

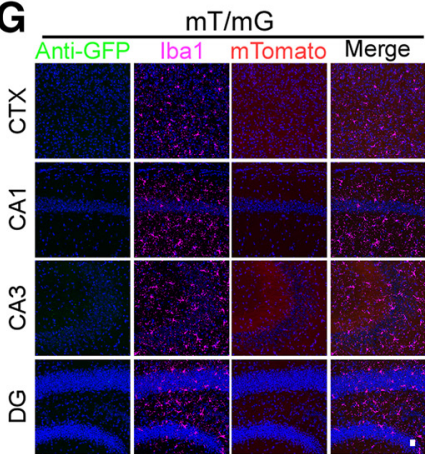

I

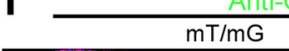

$\mathrm{C} \times 3 \mathrm{cr}^{-1}-\mathrm{CreM}^{+/-}$Rosa-Green ${ }^{\mathrm{f} / \mathrm{-}}$

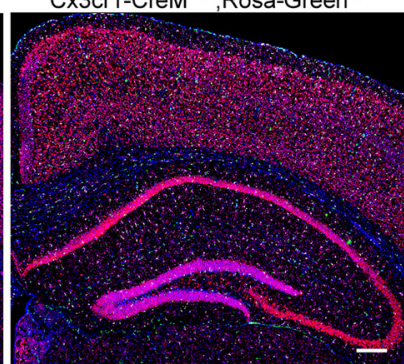

Cx3cr1-CreM ${ }^{+/-}$-Rosa-Green ${ }^{\mathrm{f} /-}$

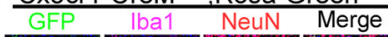

B
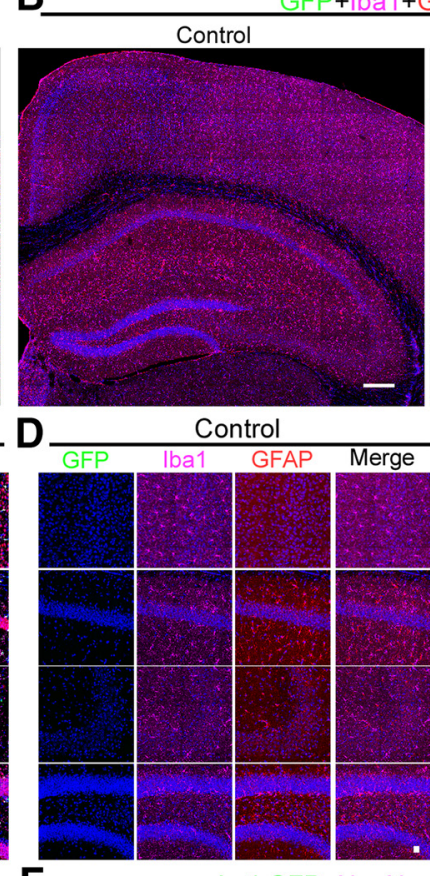

F

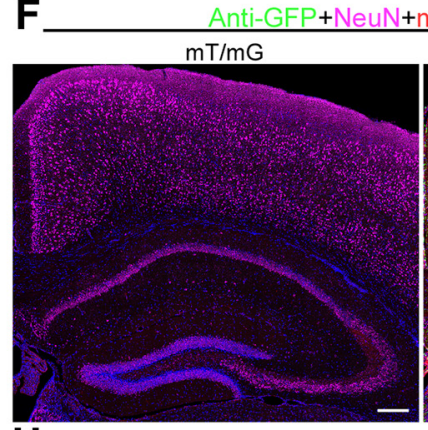

\section{$\mathbf{H}$}

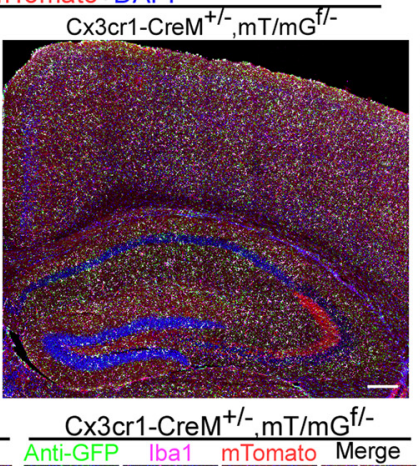

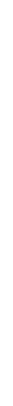

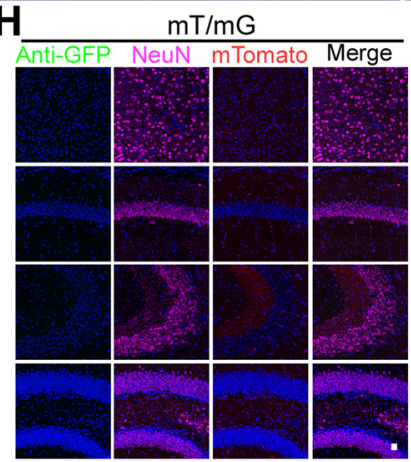

$\mathrm{mT} / \mathrm{mG}$

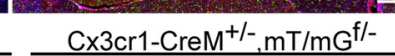

Cx3cr1-CreM ${ }^{+/-}$.Rosa-Green ${ }^{\mathrm{f} /-}$

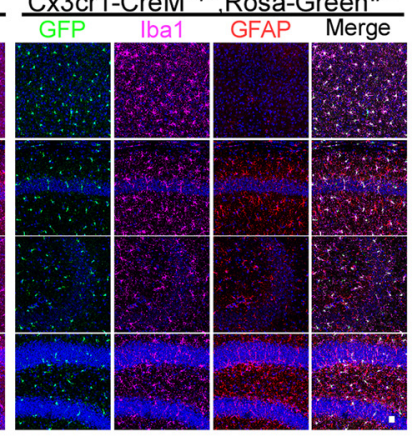

Cx3cr1-CreM ${ }^{+/-}$, Rosa-Green ${ }^{\mathrm{f} /-}$

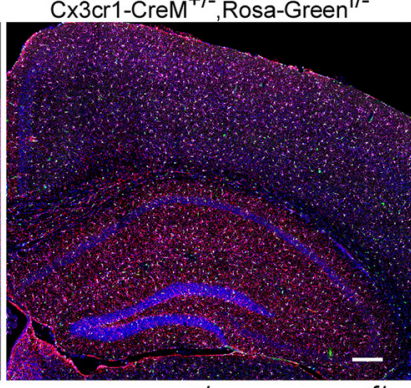

Tomato+DAPI

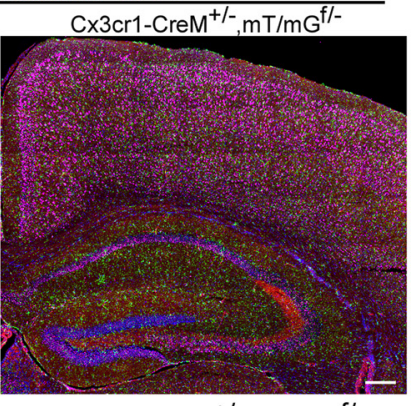

$\mathrm{C} \times 3 \mathrm{cr} 1-\mathrm{CreM}^{+/-}, \mathrm{mT} / \mathrm{mG}^{\mathrm{f} /-}$

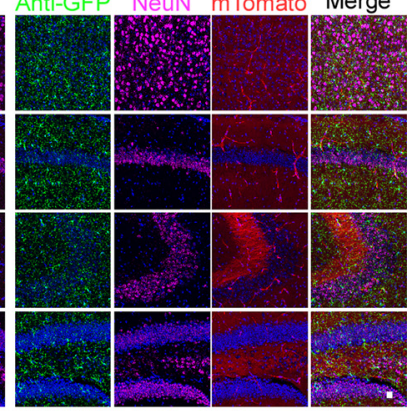

$\mathrm{C} \times 3 \mathrm{cr}^{-}-\mathrm{CreM}^{+/-} \mathrm{mT} / \mathrm{mG}^{\mathrm{f} /-}$

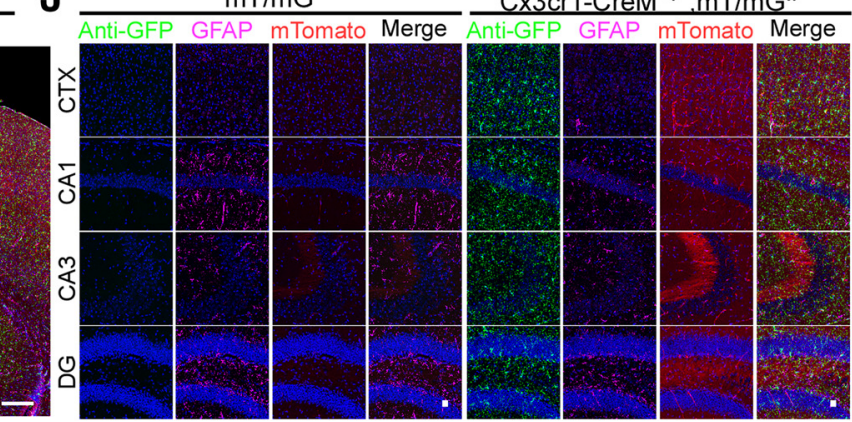

Figure 2. Specificity of $\mathrm{Cx} 3 \mathrm{cr} 1-\mathrm{Cre}$ lines in targeting microglia. $\boldsymbol{A}, \boldsymbol{B}$, Full montage of confocal images showing GFP expression in the CTX and the hippocampus of $\mathrm{Cx} 3 \mathrm{cr} 1-\mathrm{CreM} \pm$;Rosa-Green ${ }^{\mathrm{f} /-}$ mice. Brain sections were triple-stained with goat anti-lba1 (pink), rabbit 
continued

anti-NeuN (red) or chicken anti-GFAP (red), and DAPI (blue). Scale bar, $200 \mu \mathrm{m}$. C, $\boldsymbol{D}$, High-magnification images from CTX, CA1, CA3, and DG. Scale bar, $20 \mu \mathrm{m}$. $\boldsymbol{E}, \boldsymbol{F}, \boldsymbol{I}$, Full montage of confocal images showing GFP expression in the CTX and the hippocampus of $\mathrm{mT} / \mathrm{mG}^{\mathrm{f} /-}$ and $\mathrm{Cx} 3 \mathrm{cr} 1-\mathrm{Cre} \pm ; \mathrm{mT} / \mathrm{mG}^{\mathrm{f} /-}$ mouse brains. Mouse brain sections were stained with rabbit anti-GFP (green), goat anti-lba1 (pink) or mouse anti-NeuN (pink) or chicken anti-GFAP (Pink), and DAPI (blue). Scale bar, $200 \mu \mathrm{m}$. G-J, High-magnification images from CTX, CA1, CA3, and DG. Scale bar, $20 \mu \mathrm{m}$. Also see Extended Data Fig. 1-3, Fig. 2-1.

contribute to the leakage into neurons and other cells that was noted by others (Zhang et al., 2018).

In the tdTomato mouse line, we observed cre recombinase-independent leakage predominantly in the hippocampus, which is consistent with what is described at The Jackson Laboratory website, that the donating investigator reported a low level of fluorescent activity in this line. Of note, occasionally, a small fraction of tdTomato mice displayed very broad spontaneous leakage. The entire mouse body can turn pinkish in color. There- fore, caution is needed when implementing this reporter line.

Among the three fluorescent reporters tested, the $\mathrm{mT} / \mathrm{mG}$ reporter line appears to consistently deliver GFP reporter expression in a cre-dependent manner, not only in the present study, but also in many other studies (Zhao et al., 2018). The exact mechanism of spontaneous leakiness of the fluorescent reporter lines seen in the RosaGreen $^{f / f}$ and tdTomato ${ }^{f / f}$ lines remains unclear. However, given the variable leakiness of fluorescent reporter lines, it

A

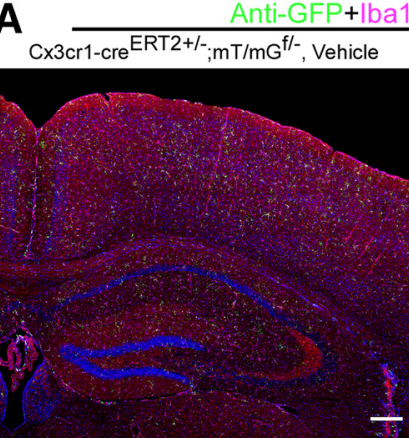

$+\mid b a 1+m$ Tomato+DAPI Cx3cr1-cre ${ }^{\mathrm{ERT} 2+/-} ; \mathrm{mT/mG}{ }^{\mathrm{f} /-}$, Tamoxifen
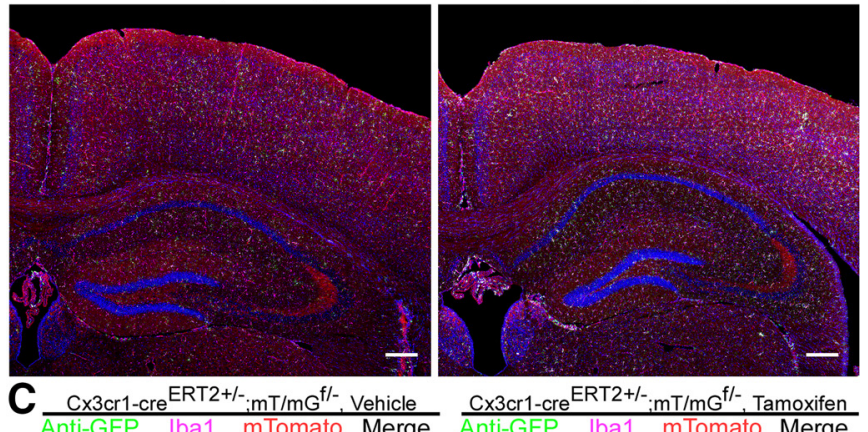

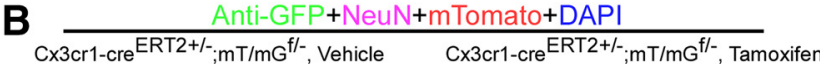
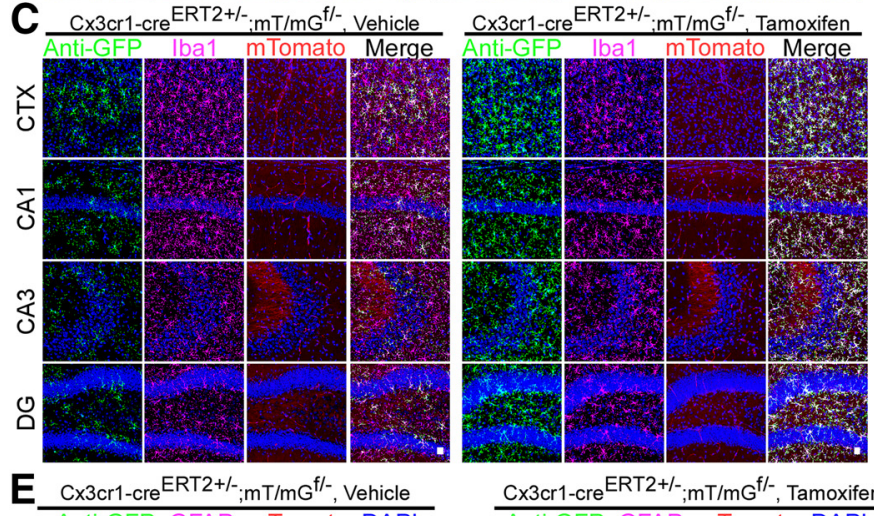

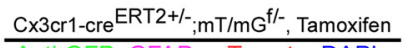
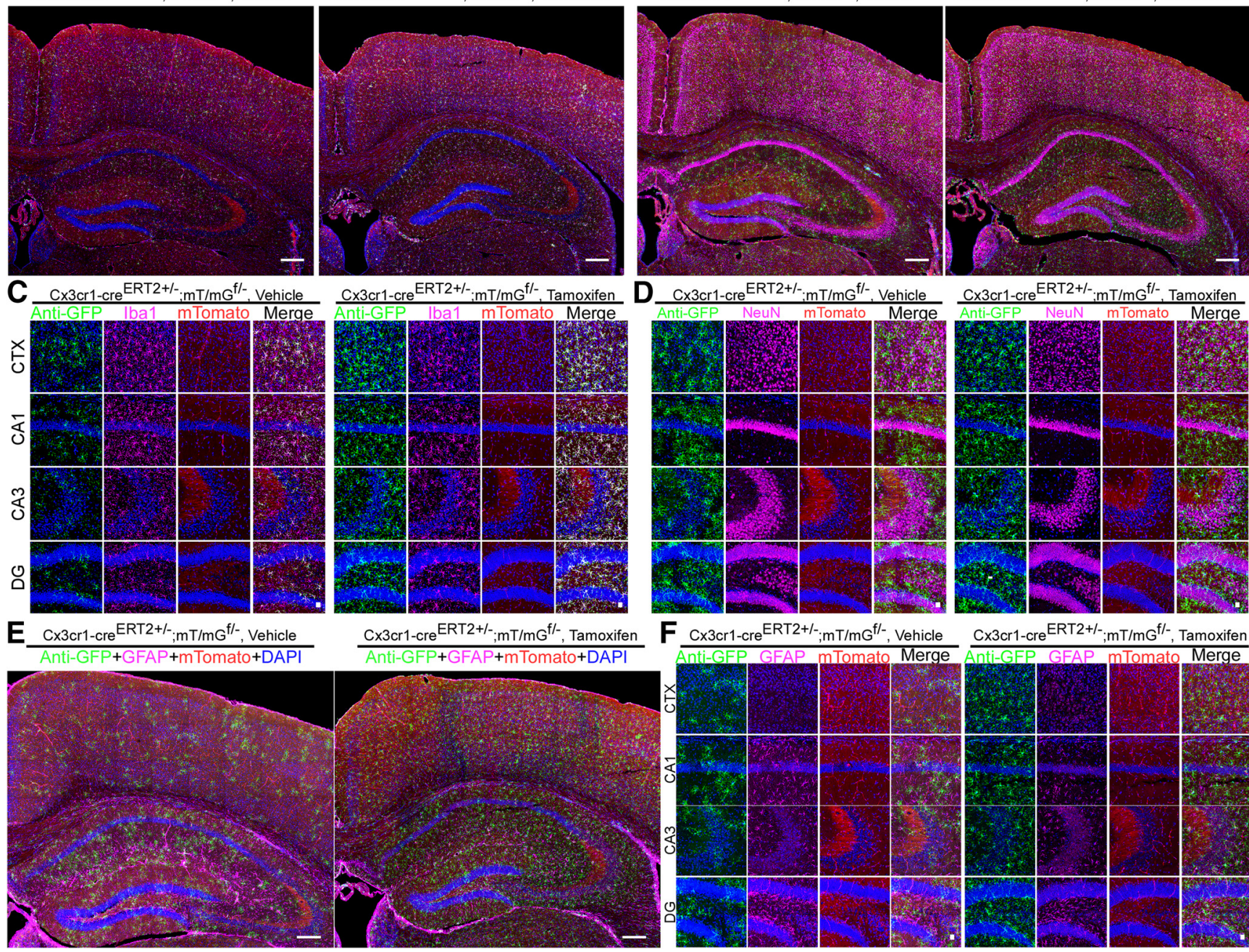

D. $\frac{\mathrm{C} \times 3 \mathrm{cr} 1-\mathrm{cre} \mathrm{ERT}^{\mathrm{ER}+-}: \mathrm{mT/mG}{ }^{\mathrm{f} /-} \text {. Vehicle }}{\text { Anti-GFP NeuN mTomato Merge }}$
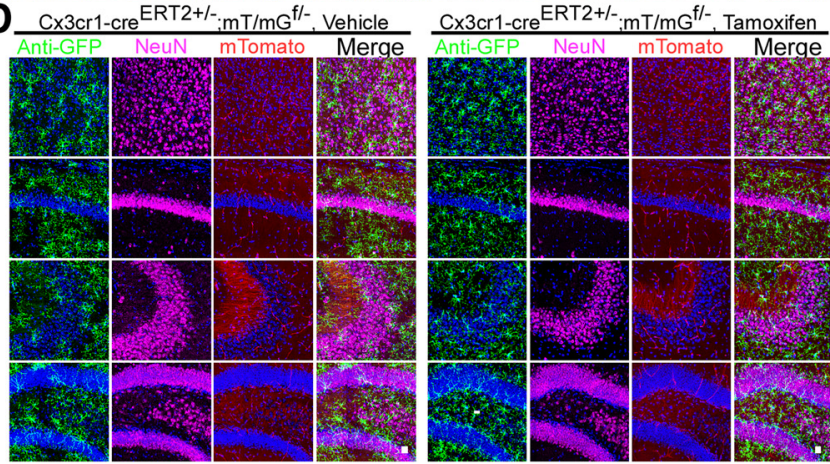

F $\mathrm{Cx3cr1- \textrm {cre } ^ { \mathrm { ERT } 2 + / - } ; \mathrm { mT } ^ { \mathrm { m } } \mathrm { mG } ^ { \mathrm { f } / - } \text { , Vehicle }}$
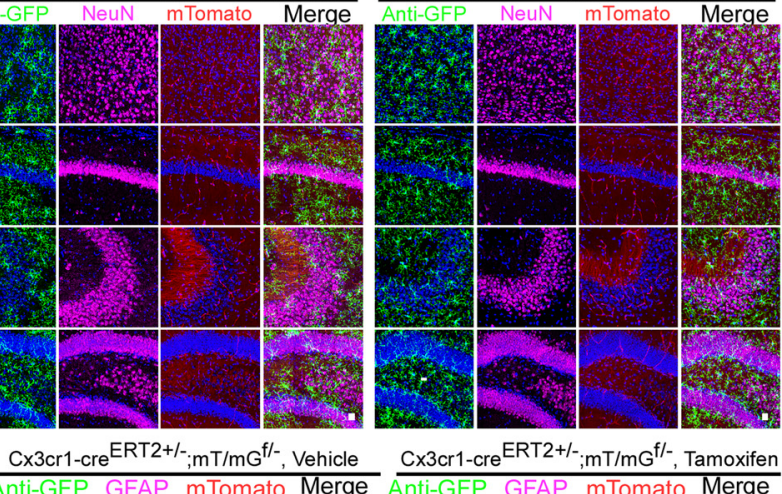

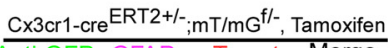
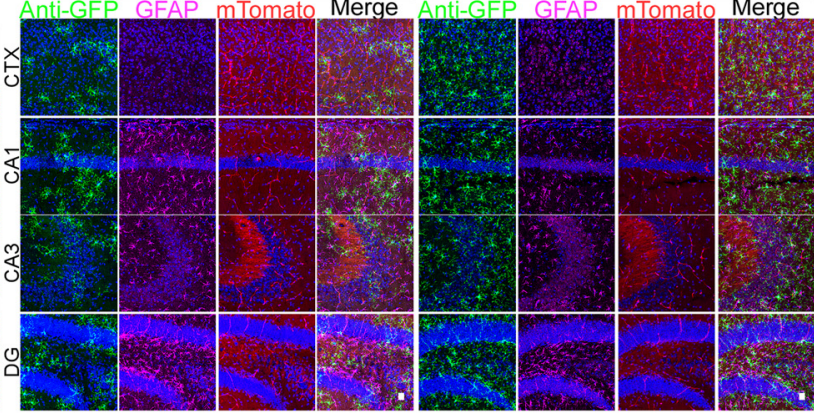

Figure 3. Basal expression of cre recombinase activity and microglial specificity of $\mathrm{Cx} 3 \mathrm{cr} 1-\mathrm{CreERT2}$ inducible line. $\boldsymbol{A}, \boldsymbol{B}, \boldsymbol{E}$, Full montage of confocal images showing GFP reporter expression in the CTX and hippocampus of Cx3cr1-CreERT2 \pm ;mTmG ${ }^{f /-}$ mice $^{-}$ treated either vehicle or tamoxifen. Mouse brain sections were stained with rabbit anti-GFP (green), goat anti-lba1 (pink) or mouse anti-NeuN (pink) or chicken anti-GFAP (Pink), and DAPI (blue). Scale bar, $200 \mu \mathrm{m}$. C, D, F, High-magnification images from CTX, CA1, CA3, and DG. Scale bar, $20 \mu \mathrm{m}$. Also see Extended Data Fig. 1-3. 
A

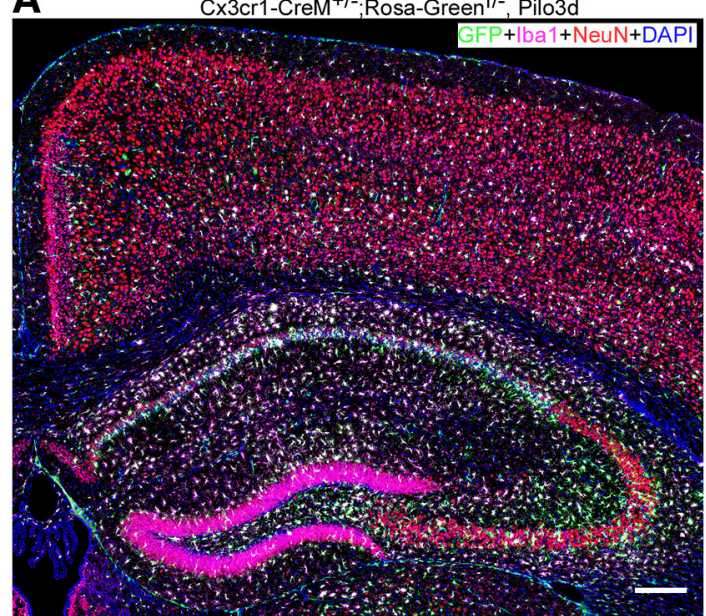

C

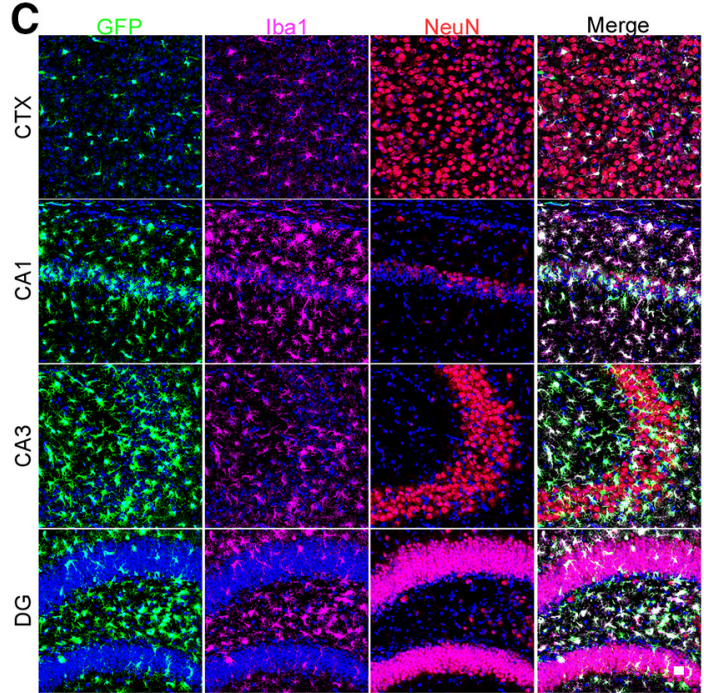

E

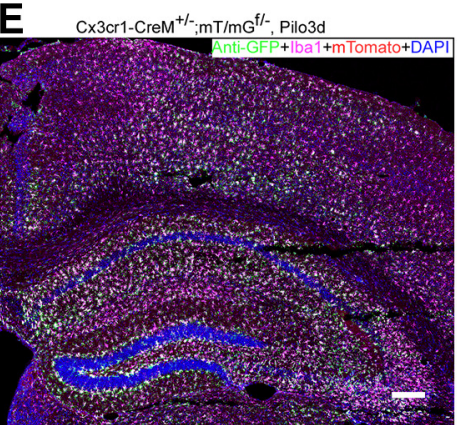

H

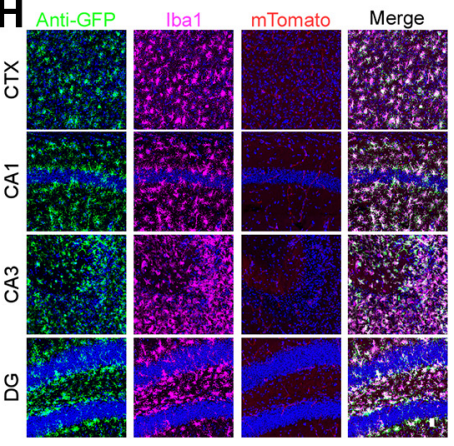

$\mathbf{F}$
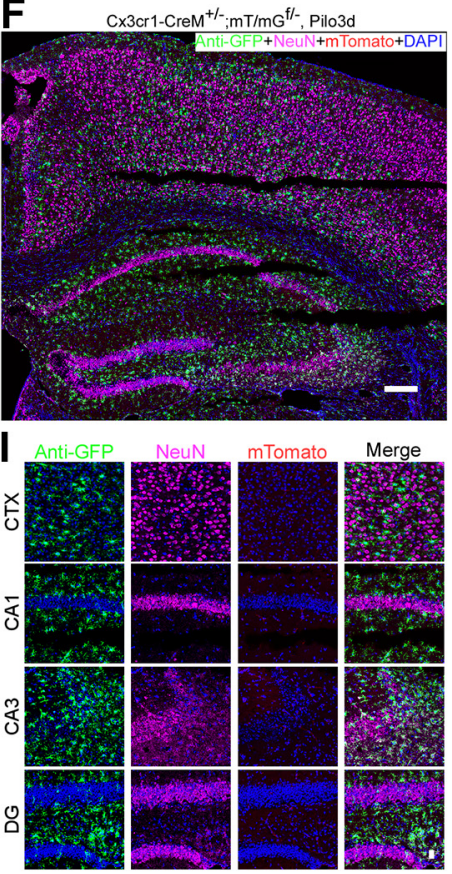

Cx3cr1-CreM ${ }^{+/-} ;$Rosa-Green ${ }^{\mathrm{f} /-}$, Pilo3d
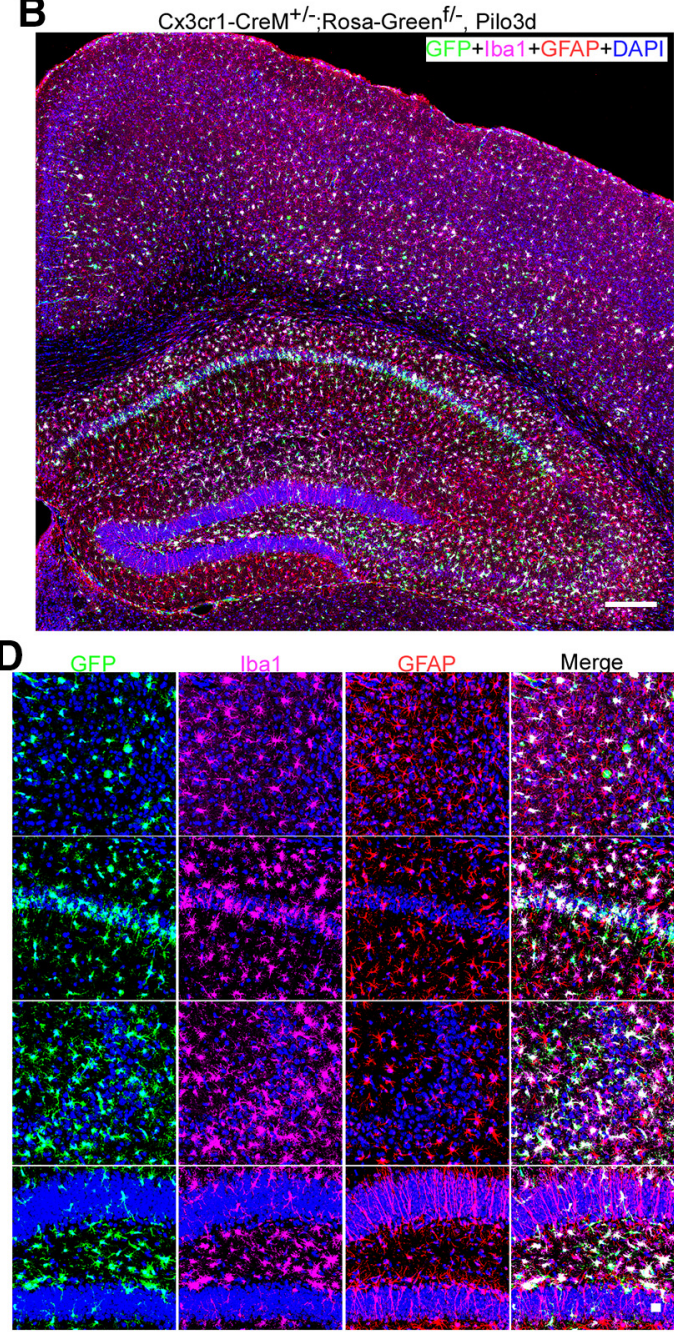

G

G $\quad$ Cx3cr1-CreM ${ }^{+/-} ; \mathrm{mT} / \mathrm{mG}^{\mathrm{f} /-}$, Pilo3d
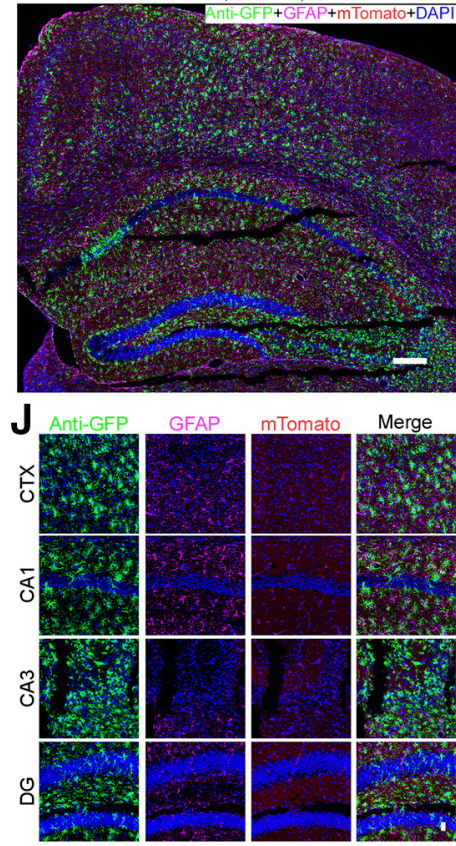

Figure 4. Specificity of Cx3cr1-Cre lines following SE. $\boldsymbol{A}, \boldsymbol{B}$, Full montage of confocal images showing GFP reporter expression in the CTX and hippocampus of Cx3cr1-Cre \pm ;Rosa-Green ${ }^{f /-}$ mice $3 \mathrm{~d}$ post-SE induced by pilocarpine. Mouse brain sections were stained 
continued

with goat anti-Iba1 (pink), rabbit anti-NeuN (red) or chicken anti-GFAP (red), and DAPI (blue). Scale bar, $200 \mu \mathrm{m}$. $\boldsymbol{C}$, $\boldsymbol{D}$, Highmagnification images from CTX, CA1, CA3, and DG. Scale bar, $20 \mu \mathrm{m}$. E-G, Full montage of confocal images showing GFP reporter expression in the CTX and hippocampus of $\mathrm{C} \times 3 \mathrm{cr} 1-\mathrm{Cre} \pm ; \mathrm{mT}_{\mathrm{mG}} \mathrm{mG}^{\mathrm{f}-}$ mice $3 \mathrm{~d}$ post-SE induced by pilocarpine. Mouse brain sections were stained with rabbit anti-GFP, goat anti-lba1 (pink) or mouse anti-NeuN or chicken anti-GFAP, and DAPI (blue). Scale bar, 200 $\mu \mathrm{m} . \boldsymbol{H}-\boldsymbol{J}$, High-magnification images from CTX, CA1, CA3, and DG. Scale bar, $20 \mu \mathrm{m}$. Also see Extended Data Fig. 1-3.

is advisable to include the reporter line(s) as a control, with a thorough analysis.

According to the description on The Jackson Laboratory website, the constitutive $\mathrm{C} \times 3 \mathrm{cr} 1-\mathrm{Cre}$ line created by Jung et al. (2000), displays leakiness into neurons when crossed with a yellow fluorescent protein (YFP) reporter line. No data were provided. The leakiness of this Cx3cr1Cre line was recently reported, but in conjunction with a Rosa-Green ${ }^{\mathrm{f} / \mathrm{f}}$ reporter line (Zhang et al., 2018). Given that the Rosa- Green $^{f / f}$ reporter line itself has spontaneous leakiness, this complicates the data interpretation. The YFP reporter line was used to verify two inducible Cx3cr1CreERT2 lines (Jung et al., 2000; Parkhurst et al., 2013). No leakiness was reported in those studies. If the YFP reporter line has no spontaneous leakiness, then the constitutive Cx3cr1-Cre line developed by Jung et al. (2000), and which is now available at The Jackson Laboratory, is not microglia specific.

In the present study, we verified that the constitutive Cx3cr1-CreM line from the MMRRC is microglia specific. We reached this conclusion based on our data from two reporter lines. Notably, this Cre-line was also deployed in a recent study by Hwang et al. (2017), who found it to drive microglial-specific expression of a reporter. However, the authors also briefly mentioned that $\sim 10 \%$ of their animals displayed significant leakage into neurons, but the data were not provided (Hwang et al., 2017). Moreover, in the same study, the GFP reporter line was created by the researchers themselves. Therefore, a thorough study of spontaneous leakiness of Hwang's reporter line is called for. It will be interesting to see whether their own GFP reporter line has any occasional spontaneous leakage.

The inducible Cx3cr1-CreERT2 mouse line that was developed by Parkhurst et al., displayed a reasonable level of microglial-specificity in their hands (Parkhurst et al., 2013). However, Zhang et al. (2018) reported that this line has significant leakage into neurons. Because the Rosa-Green ${ }^{f / f}$ reporter used by Zhang et al. (2018) displays spontaneous leakage into neurons (as we showed here), further study is required to reconcile the discrepancy. Cx3cr1-CreERT2 developed by Jung et al. (2000) was reported to drive microglia-specific expression. We found that this inducible line can drive expression of GFP reporter in a fraction of microglia before tamoxifen treatment. Thus, the cre activity in this line is not fully dependent on tamoxifen. Nevertheless, tamoxifen treatment led to broad expression of GFP reporter in microglia, without significant leakage into neurons. In the study by Zhao et al. (2018), this line was employed to deplete TSC1 in microglia. On tamoxifen treatment, a fraction of mice displayed spontaneous seizures. In contrast, no spontaneous seizures were observed in the other study (Zhang et al., 2018) that used the Cx3cr1-CreERT2 mouse line developed by Parkhurst et al. (2013). It is unclear whether this Cx3cr1-CreERT2 inducible line has cre activity leakage in the absence of tamoxifen. The partial tamoxifenindependent cre activity observed in the Cx3cr1-CreERT2 line developed by Jung et al. (2000), indicates potential embryonic deletion of TSC1 in a fraction of microglia, which may explain the differences in seizure susceptibility observed in these two studies (Jung et al., 2000; Zhang et al., 2018; Zhao et al., 2018).

All four microglia-targeting Cre lines were created using a BAC strategy, which involves insertion of a large piece of DNA containing not only the gene promoter of interest, but also many other regulatory elements that help to yield the expected expression pattern of the endogenous gene (Gong et al., 2003, 2007). A drawback of this approach is it may cause duplication of the genes brought in within the BAC constructs. This may create issues related to increased gene dosage, which could have unexpected effects, including genetic instability and ectopic gene expression. It is preferable to maintain BAC transgenic lines in a heterozygous state. This should be adopted as a standard practice when handling cre and reporter lines created with BACs.

While the $\mathrm{Cx} 3 \mathrm{cr} 1$ gene is described as being very specific to microglia in an unperturbed CNS, several studies have suggested otherwise. Meucci et al. (2000) reported that Cx3cr1is expressed in cultured neurons and promotes neuronal survival. It is unclear how relevant this is to the in vivo condition. Wang et al., reported that transient expression of $\mathrm{Cx} 3 \mathrm{cr} 1$ in neurons is neuronal protective in an ischemic model (Wang et al., 2018). However, oddly, the IHC data showed that the majority of Cx3cr1positive cells were neurons and very few, if any, were microglia. Yeo et al. (2011) reported that pilocarpinetriggered SE induced very moderate expression of $\mathrm{Cx} 3 \mathrm{cr} 1$ in astrocytes, but again the $\mathrm{IHC}$ data are puzzling. Nearly $100 \%$ of the Cx3cr1-positive cells were astrocytes. Then, where were the microglia? In the absence of a reliable anti-Cx3cr1 antibody, it is hard to draw any conclusions from these studies. In contrast, our data with reporter lines suggest there is very minimal, if any, transient Cx3cr1 activity in neurons or astrocytes following excitatory injury.

In summary, Cre lines are genetic tools essential for studying gene function in a cell-specific manner. A large array of fluorescent reporter lines has been created for cell lineage tracing and evaluating the cell specificity of many Cre lines. However, the reliability of reporter lines should not be presumed. Our study strongly suggests that both cre and reporter lines need to be thoroughly evaluated. 


\section{References}

Borges K, Gearing M, McDermott DL, Smith AB, Almonte AG, Wainer $B H$, Dingledine R (2003) Neuronal and glial pathological changes during epileptogenesis in the mouse pilocarpine model. Exp Neurol 182:21-34.

Dworzak J, Renvoisé B, Habchi J, Yates EV, Combadière C, Knowles TP, Dobson CM, Blackstone C, Paulsen O, Murphy PM (2015) Neuronal $\mathrm{C} \times 3 \mathrm{cr} 1$ deficiency protects against amyloid $\beta$-induced neurotoxicity. PLoS One 10:e0127730.

Gong S, Zheng C, Doughty ML, Losos K, Didkovsky N, Schambra UB, Nowak NJ, Joyner A, Leblanc G, Hatten ME, Heintz N (2003) A gene expression atlas of the central nervous system based on bacterial artificial chromosomes. Nature 425:917-925.

Gong S, Doughty M, Harbaugh CR, Cummins A, Hatten ME, Heintz N, Gerfen CR (2007) Targeting Cre recombinase to specific neuron populations with bacterial artificial chromosome constructs. J Neurosci 27:9817-9823.

Haimon Z, Volaski A, Orthgiess J, Boura-Halfon S, Varol D, Shemer A, Yona S, Zuckerman B, David E, Chappell-Maor L, Bechmann I, Gericke M, Ulitsky I, Jung S (2018) Re-evaluating microglia expression profiles using RiboTag and cell isolation strategies. Nat Immunol 19:636-644.

Hwang HW, Saito Y, Park CY, Blachère NE, Tajima Y, Fak JJ, Zucker-Scharff I, Darnell RB (2017) cTag-PAPERCLIP reveals alternative polyadenylation promotes cell-type specific protein diversity and shifts Araf isoforms with microglia activation. Neuron 95:1334-1349.e5.

Jung S, Aliberti J, Graemmel P, Sunshine MJ, Kreutzberg GW, Sher A, Littman DR (2000) Analysis of fractalkine receptor CX(3)CR1 function by targeted deletion and green fluorescent protein reporter gene insertion. Mol Cell Biol 20:4106-4114.

Kim I, Mlsna LM, Yoon S, Le B, Yu S, Xu D, Koh S (2015) A postnatal peak in microglial development in the mouse hippocampus is correlated with heightened sensitivity to seizure triggers. Brain Behav 5:e00403.

Mazzetti S, Frigerio S, Gelati M, Salmaggi A, Vitellaro-Zuccarello L (2004) Lycopersicon esculentum lectin: an effective and versatile endothelial marker of normal and tumoral blood vessels in the central nervous system. Eur J Histochem 48:423-428.

Meucci O, Fatatis A, Simen AA, Miller RJ (2000) Expression of CX3CR1 chemokine receptors on neurons and their role in neuronal survival. Proc Natl Acad Sci USA 97:8075-8080.

Müller CJ, Gröticke I, Bankstahl M, Löscher W (2009) Behavioral and cognitive alterations, spontaneous seizures, and neuropathology developing after a pilocarpine-induced status epilepticus in C57BL/6 mice. Exp Neurol 219:284-297.

Parkhurst CN, Yang G, Ninan I, Savas JN, Yates JR 3rd, Lafaille JJ, Hempstead BL, Littman DR, Gan WB (2013) Microglia promote learning-dependent synapse formation through brain-derived neurotrophic factor. Cell 155:1596-1609.

Peng J, Gu N, Zhou L, B Eyo U, Murugan M, Gan WB, Wu LJ (2016) Microglia and monocytes synergistically promote the transition from acute to chronic pain after nerve injury. Nat Commun 7:12029.

Racine RJ (1972) Modification of seizure activity by electrical stimulation. II. Motor seizure. Electroencephalogr Clin Neurophysiol 32:281-294.

Sahay A, Scobie KN, Hill AS, O'Carroll CM, Kheirbek MA, Burghardt NS, Fenton AA, Dranovsky A, Hen R (2011) Increasing adult hippocampal neurogenesis is sufficient to improve pattern separation. Nature 472:466-470.

Subbanna S, Nagre NN, Shivakumar M, Joshi V, Psychoyos D, Kutlar A, Umapathy NS, Basavarajappa BS (2018) CB1R-mediated activation of caspase- 3 causes epigenetic and neurobehavioral abnormalities in postnatal ethanol-exposed mice. Front Mol Neurosci $11: 45$.

Wang J, Gan Y, Han P, Yin J, Liu Q, Ghanian S, Gao F, Gong G, Tang $Z$ (2018) Ischemia-induced neuronal cell death is mediated by chemokine receptor CX3CR1. Sci Rep 8:556.

Wieghofer P, Prinz M (2016) Genetic manipulation of microglia during brain development and disease. Biochim Biophys Acta 1862:299309.

Yeo SI, Kim JE, Ryu HJ, Seo CH, Lee BC, Choi IG, Kim DS, Kang TC (2011) The roles of fractalkine/CX3CR1 system in neuronal death following pilocarpine-induced status epilepticus. J Neuroimmunol 234:93-102.

Yona S, Kim KW, Wolf Y, Mildner A, Varol D, Breker M, Strauss-Ayali D, Viukov S, Guilliams M, Misharin A, Hume DA, Perlman H, Malissen B, Zelzer E, Jung S (2013) Fate mapping reveals origins and dynamics of monocytes and tissue macrophages under homeostasis. Immunity 38:79-91.

Zhang B, Zou J, Han L, Beeler B, Friedman JL, Griffin E, Piao YS, Rensing NR, Wong M (2018) The specificity and role of microglia in epileptogenesis in mouse models of tuberous sclerosis complex. Epilepsia 59:1796-1806.

Zhao X, Liao Y, Morgan S, Mathur R, Feustel P, Mazurkiewicz J, Qian J, Chang J, Mathern GW, Adamo MA, Ritaccio AL, Gruenthal M, Zhu X, Huang Y (2018) Noninflammatory changes of microglia are sufficient to cause epilepsy. Cell Rep 22:2080-2093. 